\title{
Characterization of Aspergillus nidulans Mutants in Carbon Metabolism Isolated after D-Galacturonate Enrichment
}

\author{
By J. H. A. A. UITZETTER,† C. J. BOS AND J. VISSER* \\ Department of Genetics, Agricultural University, Gen. Foulkesweg 53, 6703 BM Wageningen, \\ The Netherlands
}

(Received 1 October 1985 ; revised 4 December 1985)

\begin{abstract}
A selective method for the isolation of Aspergillus nidulans mutants defective in the pyruvate dehydrogenase complex was devised. The essential steps in the procedure were a mutagenic treatment of conidia with X-rays to about $50 \%$ survival, followed by filtration enrichment in minimal medium with D-galacturonate as sole carbon source, and rescue on complete medium with acetate. The mutants thus isolated were phenotypically characterized on the basis of growth tests, and different genotypes were assigned on the basis of complementation tests. The majority of the mutants that were unable to utilize galacturonate were defective in one of the components of the pyruvate dehydrogenase complex. In addition, mutants defective in pyruvate carboxylase, mutants defective in glycerol catabolism and some novel mutants which were only unable to use D-galacturonate as carbon source were found. At least two genes were shown to be involved in D-galacturonate metabolism.
\end{abstract}

\section{INTRODUCTION}

Aspergillus nidulans mutants defective in carbon metabolism have been found after mutagenic treatment, with a low percentage of survival without further enrichment procedures (Skinner \& Armitt, 1972). Carbon metabolism mutants are commonly selected by a filtration enrichment technique. This method has been used to isolate both gluconeogenic (Armitt et al., 1976) and glycolytic mutants. For the isolation of the latter a disaccharide like sucrose (Payton et al., 1977) or a readily metabolized monosaccharide like glucose (Payton et al., 1977; Bos et al., 1981) were used.

We reported previously growth of $A$. nidulans on D-galacturonate as sole carbon and energy source (Uitzetter et al., 1982). Tests with mutants defective in pyruvate kinase ( $p k i)$ and the pyruvate dehydrogenase complex ( $p d h$ ) showed good growth of the former but no growth of the latter mutants on this substrate. This indicates a metabolic pathway for D-galacturonate leading to pyruvate as one of the degradation products. Such a pathway exists in Escherichia coli (Ashwell et al., 1958). Payton et al. (1977) isolated eight pdh mutants among some 200 mutants selected for their inability to grow on sucrose. They used $N$-methyl- $N^{\prime}$-nitro- $N$-nitrosoguanidine as a mutagen at a $0.5 \%$ survival level.

Genetic analysis of these $p d h$ mutants showed that the genes $p d h A$ and $p d h B$ belong to linkage groups I and V respectively. Bos et al. (1981) reported the isolation of a number of other pyruvate dehydrogenase complex mutants which were obtained by UV-treatment at a $75-80 \%$ survival rate followed by enrichment in glucose minimal medium. Of the 160 strains isolated, $54 \%$ had defective pyruvate dehydrogenase complex activity. Among these mutants a third distinct gene, $p d h C$, was found, and assigned to linkage group VIII. In order to obtain other mutants defective

$†$ Present address: Netherlands Organization for the Advancement of Pure Research (ZWO), The Hague, The Netherlands.

Abbreviations: CM, complete medium; MM, minimal medium. 
in pyruvate metabolism, D-galacturonate was included in the medium for enrichment. This paper describes the results of an X-ray mutation experiment with $A$. nidulans followed by Dgalacturonate enrichment, and the characterization of mutant classes found.

\section{METHODS}

Strains. The Aspergillus nidulans strain used for mutation was WG145 (wA3 pyroA4). This strain has wild-type characteristics for carbon source utilization. For other experiments the following strains were used: WG001 ( $y A 2$ phenA2 lysB5); WG203 (yA2 meaA3 pA1 sB3 nicB8 riboB2); WG097 (pabaA1 yA2 pdhAl); WG100 (pabaAl yA2 pdhBl); WG107 (pabaAl yA2 pdhCl); WG102 (pabaAl yA2 pycA2); WG135 (pabaAl yA2 pycB4); WG196 (pabaAl yA2 glcAl) and WG197 (pabaAl yA2 glcB33). These strains were derived from the original Glasgow strains of $A$. nidulans (Pontecorvo et al., 1953). All symbols have their usual meaning (Clutterbuck, 1973; Payton, 1978). For mutants concerned with D-galacturonate metabolism the gene symbol gaa is used.

Media and growth tests. A. nidulans WG 145 was grown on complete medium (CM) according to Pontecorvo et al. (1953) except that glucose was replaced by acetate $(0.1 \mathrm{M})+$ sucrose $(0.01 \mathrm{M})$. Growth tests were done on minimal medium (MM) supplemented with pyridoxine. $\mathrm{HCl}\left(1 \mathrm{mg} \mathrm{l}^{-1}\right)$. The medium was solidified with $1.5 \%(\mathrm{w} / \mathrm{v})$ agar. Unless stated otherwise glucose was omitted and other carbon sources were added instead. Carbon sources ( $\mathrm{pH}$ 6.0) were added separately as membrane filter sterilized solutions.

The final concentrations of the carbon sources were: D-galacturonate $0.05 \mathrm{M}$; D-gluconate $0.05 \mathrm{M}$; D-glucose $0.05 \mathrm{M}$; glycerol $0.05 \mathrm{M} ; \mathrm{D}-\mathrm{xylose} 0.05 \mathrm{M}$; acetate $0.1 \mathrm{M}$; acetate $0.1 \mathrm{M}+$ sucrose $0.01 \mathrm{M}$; L-glutamate $0.05 \mathrm{M}+\mathrm{D}$ fructose $0.01 \mathrm{M} ;$ glycerol $0.05 \mathrm{M}+$ acetate $0.1 \mathrm{M}$. All acids were used as their sodium salts. The growth of the wildtype and mutant strains was scored after $2 \mathrm{~d}$ incubation at $37^{\circ} \mathrm{C}$.

Induction and isolation of mutants. Mutations were induced by X-ray treatment for $6.5 \mathrm{~min}$ at a dose yielding $51 \%$ survival, using a Philips Röntgen apparatus equipped with a MCN 161 tube $(160 \mathrm{kV}, 19 \mathrm{~mA}, 2 \mathrm{~mm}$ Al-filter) placed $36 \mathrm{~cm}$ from the bottom of a Petri dish which contained $15 \mathrm{ml}$ of a conidial suspension of strain WG145 (about $5 \times 10^{7}$ conidia $\mathrm{ml}^{-1}$ ). The total dose was $2.5 \mathrm{krad}$. Survival was determined before and after irradiation by plating a diluted suspension of conidia on CM acetate. Irradiated conidia were transferred to $300 \mathrm{ml}$ liquid MM in a l litre infusion bottle with D-galacturonate as carbon source. The flask was incubated in a reciprocal shaker bath ( 160 strokes $\mathrm{min}^{-1}$ ) at $37^{\circ} \mathrm{C}$ for $12 \mathrm{~h}$. Conidia that did not germinate were separated from hyphae by filtration over cotton wool; the conidia were then collected on a membrane filter and incubated in $100 \mathrm{ml}$ fresh medium for a further $11 \mathrm{~h}$. Finally the conidia that had still not germinated and that had passed a second filtration procedure were resuspended in $0.5 \mathrm{ml} 0.8 \%(\mathrm{w} / \mathrm{v}) \mathrm{NaCl}$. After appropriate dilution the suspension was plated at a density of about 60 colonies per plate in $\mathrm{CM}$ with acetate and incubated at $37^{\circ} \mathrm{C}$ for $2 \mathrm{~d}$. From the colonies thus obtained master plates were made on $\mathrm{CM}$ with acetate and replicas were made on $\mathrm{MM}$ with D-galacturonate and acetate respectively to identify carbon metabolism mutants. Mutants which showed leaky growth or no growth on Dgalacturonate were then purified by plating conidia from a single mutant colony. Monospore cultures were made and conidia were preserved on dehydrated silica gel according to Perkins (1962).

Phenotypic classification. To characterize the mutants by their phenotype, growth tests were done as described above. Criteria to assign phenotypes are shown in Table 1.

Complementation tests. To assign mutant genes, $p d h A, p d h B$ and $p d h C$, and $p y c A$ pycB tester strains with different colour and vitamin markers were used for complementation. Heterokaryotic mycelium was obtained according to Pontecorvo et al. (1953). Twin pieces of heterokaryotic mycelium were transferred to MM with sucrose and to MM with acetate + sucrose. The latter medium gave much better growth than acetate alone. Complementation with $g l c$ mutants was done with diploids constructed with the $g l c A l$ and $g l c B 33$ strains respectively. Growth tests with diploids were done on MM with glycerol and galacturonate respectively, using $\mathrm{MM}$ with sucrose as a control.

Enzyme assays. Uronic acid isomerase (EC 5.3.1.12), D-altronic acid dehydrogenase (EC 1.1.1.58) and Daltronic acid hydrolyase (EC 4.2.1.7) were assayed according to Ashwell (1962).

Chemicals. D-Galacturonic acid was obtained from Aldrich. Tagaturonic acid was synthesized according to Ehrlich \& Guttmann (1934); altronic acid was synthesized according to Pratt \& Richtmeyer (1955). These acids were a gift from the Department of Organic Chemistry, Agricultural University, Wageningen, The Netherlands. All other chemicals were obtained from Merck.

\section{RESULTS AND DISCUSSION}

The survival of the irradiated conidia was $51 \%$. In order to prevent background damage a relatively low dose rate was used in this mutation experiment. The mutagenesis and enrichment procedure resulted in 2232 colonies, of which 840 were tested for their ability to grow on Dgalacturonate. From these, $209(25 \%)$ showed little or no growth on this carbon source and were 
Table 1. Growth criteria used to assign phenotypes to isolated A. nidulans mutants

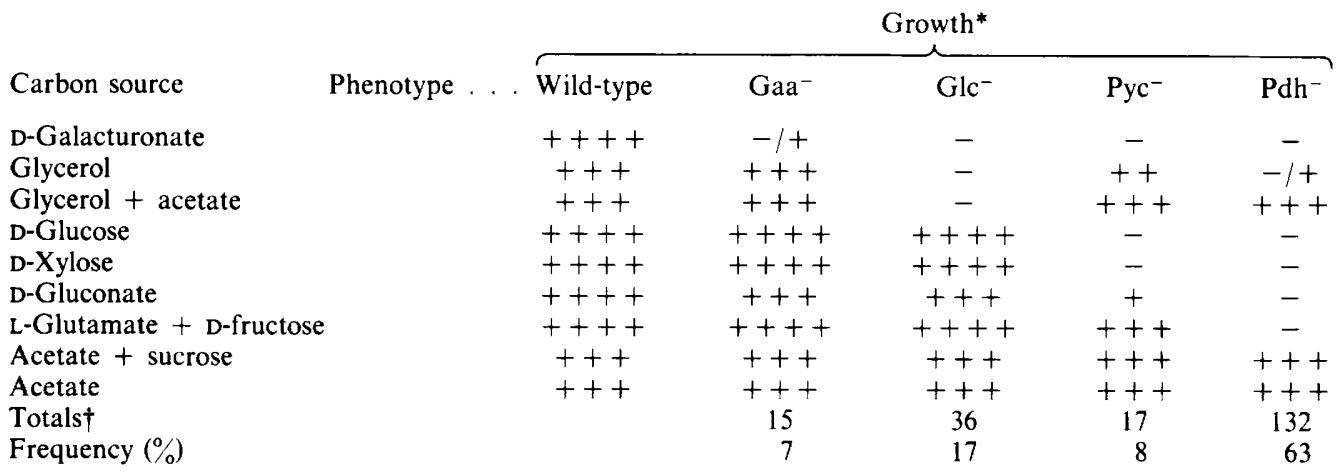

* ++++ , good; +++ , moderate; ++ , poor; + , very poor; and - , no growth.

$\dagger$ Nine strains could not be assigned with these criteria.

therefore of the desired phenotype; $145(17 \%)$ appeared to have other deficiencies and were not considered further. Table 1 summarizes the criteria which were used to assign different phenotypes to the D-galacturonate non-utilizing or ill-utilizing mutants. Growth behaviour on the various substrates was discussed by Uitzetter et al. (1982).

The $\mathrm{Pdh}^{-}, \mathrm{Pyc}^{-}$and $\mathrm{Glc}^{-}$phenotypes classified by these growth tests were then shown by complementation to correspond with genotypes already known; their frequencies are shown in Table 1. Mutants in glycerol metabolism $(\mathrm{glc})$ and D-galacturonate metabolism $(\mathrm{gaa})$ showed normal sporulation like the parental strain, in contrast to $p d h$ and $p y c$ mutants. The number of mutants recovered was very high. This could have been due to a relatively quick growth of the mycelium which made the enrichment period sufficiently short to a void loss of mutants by crossfeeding and aggregation. Only nine mutants could not be assigned by the phenotypic characterization.

\section{Pyruvate dehydrogenase complex ( $p d h)$ mutants}

The majority $(63 \%)$ of the isolated mutants turned out to have a defective pyruvate dehydrogenase complex. Complementation of these mutants was done with the three marker genes known for this complex. pdhA is the structural gene for lipoate acetyltransferase $\left(\mathrm{E}_{2}\right)$, and $p d h C$ and $p d h B$ are the structural genes for the two subunits of pyruvate decarboxylase $\left(\mathrm{E}_{1}\right)$. The distribution of the $p d h$ mutants over the three genes was $37 \% p d h A, 28 \% p d h B$ and $35 \% p d h C$. It is obvious that other selection techniques are required to find mutants with defects in structural genes coding for the other complex components, viz. lipoamide dehydrogenase $\left(\mathrm{E}_{3}\right)$ and the two regulating enzymes pyruvate dehydrogenase kinase and pyruvate dehydrogenase-phosphatase. The pyruvate dehydrogenase kinase, which is firmly bound to the core of the complex, inactivates the complex by phosphorylating the $\alpha$ subunit of $E_{1}$. A specific phosphatase, which is loosely associated with the complex, reactivates the enzyme (Hucho et al., 1972).

A possible reason for missing the $\mathrm{E}_{3}$ mutants of this complex might be the occurrence of more than one gene coding for $E_{3}$. For instance, in mammalian tissues several lipoamide dehydrogenase isoenzymes have been found which are functionally exchangeable between the pyruvate and 2-oxoglutarate dehydrogenase multienzyme complexes (Kenney et al., 1972; Sakurai et al., 1970). Of course, pdh kinase mutants will not be found as the complex will be fully active under those circumstances. In the case of $p d h$-phosphatase mutants a partially active complex may still be present since phosphorylation is a post-translational event. This will result in loss of such mutants in an early stage of the enrichment. Also it is not known whether nonspecific phosphatase activities present act to some extent on the phosphorylated pyruvate dehydrogenase complex. 


\section{Glycerol metabolism ( $\mathrm{glc}$ ) mutants}

All the $g l c$ mutants isolated belonged to a single class as shown by complementation in diploids; they were identified as $g l c B$. It was necessary to construct diploids for complementation analysis because heterokaryons showed very poor growth, if any, on glycerol (McCullough et al., 1977). glc mutants were first isolated by Payton (1978) after mutagenic treatment followed by enrichment in glycerol medium. He assigned two genes: glc $A$ (linkage group $\mathrm{V}$ ) and $g l c B$ (linkage group I). The $g l c A$ mutant showed very poor growth on glycerol. The glcB mutant was absolute, and its growth on other carbon sources in the presence of glycerol was strongly inhibited. The exact nature of the $g l c A$ and $g l c B$ mutants is still unresolved. The glc $B$ mutant was suspected to be deficient in glycerol-3-phosphate dehydrogenase because of the interference of glycerol with growth on other carbon sources, which is also observed in E. coli (Cozzarelli et al., 1965) and Salmonella typhimurium (Aceves-Pina et al., 1974). We were unable, however, to come to this conclusion since we never detected a glycerol kinase activity in these fungal extracts, which makes it unlikely that $g l c B$ corresponds to a $s n$-glycerol-3-phosphate dehydrogenase defect. Recently, we have found a NADP ${ }^{+}$-dependent glycerol dehydrogenase activity as well as a weak glyceraldehyde kinase activity in crude extracts of wild-type $A$. nidulans mycelium ( $J$. Visser and co-workers, unpublished results) which suggests another pathway. Both $g l c A$ and $g l c B$ failed to grow on $\mathrm{D}$-galacturonate, indicating that glycerol and D-galacturonate catabolism have at least one step in common.

\section{Pyruvate carboxylase (pyc) mutants}

Skinner \& Armitt (1972) were the first to describe pyc mutants after mutagenic treatment of $A$. nidulans conidia to $1 \%$ survival. They tested 8000 colonies of which 3 among 55 sucrose-negative survivors had the $p y c$ genotype. A large number of $p y c$ mutants have since been isolated by Payton et al. (1977) and Bos et al. (1981). Complementation data suggest intragenic complementation, although the existence of two closely linked genes is not completely excluded (C. J. Bos, unpublished results).

In this experiment 17 mutants showed a $\mathrm{Pyc}^{-}$phenotype (Table 1). They were completely unable to use D-galacturonate for growth, in contrast to the pyc mutants isolated earlier (Bos et al., 1981). The pyc genotype of these mutants was confirmed by complementation which occurred only with one of the two pyc test strains (WG102 and WG135).

\section{D-Galacturonate metabolism (gaa) mutants}

In the mutation experiment described, 15 mutants were isolated which were exclusively unable to use D-galacturonate as sole carbon and energy source. These mutants showed normal growth on the other carbon sources listed in Table 1, indicating that D-galacturonate was not catabolized through one of the main pathways for carbohydrate degradation. Apart from the carbon sources already mentioned the following compounds were tested for growth; D-gluconate, D-glucarate, D-glucuronate, D-altronate, D-galactarate and D-tagaturonate. All compounds except the latter two were able to support growth both in wild-type and gaa mutants.

In E. coli a uronic acid catabolic pathway has been demonstrated (Ashwell et al., 1958). After an initial isomerization reaction, a dehydrogenation leads to $D$-altronate. The next steps involve dehydration of the last intermediate to 2-keto-3-deoxy-gluconic acid and subsequent phosphorylation. Finally an aldolase reaction leads to pyruvate and glyceraldehyde 3-phosphate as end products. In preliminary experiments with the wild-type strain of $A$. nidulans grown on D-galacturonate none of the reactions mentioned above could be detected, either in cell-free extracts, or as membrane-bound activity. Therefore, another D-galacturonate degradation pathway is likely to exist in this fungus. Alternative routes have been observed in Pseudomonas spp. (Kilgore \& Starr, 1959; Preiss \& Ashwell, 1963a, b), and in Agrobacterium tumefaciens (Chang \& Feingold, 1969), where oxidation of D-galacturonate to D-galactarate is the initial step. The specific pathway in $A$, nidulans is unknown, but from the growth characteristics of $p d h, p y c$ and pki mutants it is clear that pyruvate is one end-product. On the basis of the phenotype of the glc $A$ and $g l c B$ mutants D-glyceraldehyde is suggested to be the intermediate which the two routes have in common. This implies a non-phosphorylative breakdown route of $D$-galacturonate in this fungus. 
Table 2. Allocation of gaaA and gaaB by mitotic haploidization of diploids gaaA/WG203 and gaaB/WG203

\begin{tabular}{|c|c|c|c|c|c|c|}
\hline & & & & No. & loids & \\
\hline & Mutant strain & Tester strain & gaaAl & $\mathrm{gaaA}^{+}$ & $g a a B 2$ & $\mathrm{gaaB}^{+}$ \\
\hline I & $y A^{+}$ & & 0 & 0 & 4 & 1 \\
\hline & & $y A 2$ & 20 & 20 & 0 & 39 \\
\hline II & $w A$ & & 12 & 29 & 25 & 90 \\
\hline & & $w A^{+}$ & 20 & $\cdot 20$ & 4 & 39 \\
\hline III & $m e A^{+}$ & & 0 & 3 & * & * \\
\hline & & meA3 & 32 & 46 & * & * \\
\hline IV & pyroA4 & & 15 & 14 & 13 & 57 \\
\hline & & pyroA & 17 & 35 & 16 & 72 \\
\hline V & $p A^{+}$ & & * & * & 13 & 58 \\
\hline & & $p A I$ & $*$ & * & 16 & 71 \\
\hline VI & $s B^{+}$ & & 32 & 1 & 16 & 53 \\
\hline & & $s B 3$ & 0 & 48 & 13 & 76 \\
\hline VII & nic $B^{+}$ & & 17 & 25 & 9 & 77 \\
\hline & & nic $B 8$ & 15 & 24 & 20 & 52 \\
\hline VIII & ribo $B^{+}$ & & 20 & 20 & 13 & 56 \\
\hline & & riboB2 & 12 & 29 & 16 & 73 \\
\hline
\end{tabular}

\section{Existence and allocation of different gaa genes}

In order to allocate gaaA a heterozygous diploid was constructed with master strain WG203, and haploid segregants were induced with $p$-fluorophenylalanine according to McCully \& Forbes (1965).

From the results shown in Table 2 it can be concluded that the gaaA gene belongs to linkage group VI, since genes on this chromosome remain together during mitotic haploidization. Because all gaa strains isolated were in the wA3 pyroA4 background one of the haploid segregants was used for complementation experiments with the other gaa mutants isolated. Heterokaryons were tested on MM with sucrose and MM with galacturonate respectively. The gaaAI strain was able to complement with all other gaa mutants, but not with the parental strain from which it was derived.

One of the other gaa mutants ( $g a a B 2$ ) was also analysed in a heterozygous diploid with the same master strain (Table 2). Because a conclusive assignment of gaaB was impossible, a second heterozygous diploid was constructed with WG001 in order to examine the segregation of markers on chromosome I, III and V (results not shown). The results indicated that gaaB is located on linkage group I which confirms that at least two genes exist coding for enzymes in D-galacturonate metabolism.

\section{Conclusions}

D-Galacturonate is a very suitable substrate for enrichment of mutants defective in galacturonate, glycerol and pyruvate metabolism. The defined classes of mutants thus derived undoubtedly give a tool for resolving the subsequent steps of both D-galacturonate and glycerol metabolism. At least two genes, gaaA and gaaB, are involved in D-galacturonate breakdown. They are located on linkage groups VI and I respectively. Since pki mutants grew on D-galacturonate whereas $p d h$ and $p y c$ mutants did not, this indicated that galacturonate is degraded to a metabolite beyond phosphoenolpyruvate, viz. pyruvate. In E. coli D-galacturonate degradation finally leads to D-glyceraldehyde 3-phosphate and pyruvate as end-products. The isolation of a large number of $g l c B$ mutants by galacturonate enrichment indicates that in $A$. nidulans glycerol catabolism has at least one step in common with D-galacturonate breakdown. Since dehydrogenation of glycerol seems to be the initial step in the utilization of this substrate by $A$. nidulans it is postulated that D-glyceraldehyde is an intermediate in both pathways. Detailed studies on glycerol metabolism are in progress, but thus far we have not been able to identify which enzyme function is coded by the $g l c B$ gene. 
We are indebted to Mr J. Baars and Mr J. F. Eikelenstam (ITAL, Wageningen) for performing the X-ray treatment, to Mrs G. Kobus-Coops for the analysis of diploids and to Dr C. F. Roberts (University of Leicester, UK) for providing the $g / c A I$ and $g l c B 33$ strains. We thank Prof. dr Ae. de Groot for his collaboration on the synthesis of D-tagaturonate and D-altronate.

\section{REFERENCES}

Aceves-Pina, E., Ortega, M. V. \& Artis, M. (1974). Linkage of the Salmonella typhimurium chromosomal loci encoding for the cytochrome linked $\mathrm{L}-\alpha$-glycerophosphate dehydrogenase and amylomaltase activities. Archives of Microbiology 101, 59-70.

Armitt, S., McCullough, W. \& Roberts, C. F. (1976). Analysis of acetate non-utilizing ( $a \mathrm{cu})$ mutants in Aspergillus nidulans. Journal of General Microbiology 92, 263-282.

ASHWELL, G. (1962). Enzymes of glucuronic and galacturonic acid metabolism in bacteria. Methods in Enzymology 5, 190-208.

Ashwell, G., Wahba, A. J. \& Hickman, J. (1958). A new pathway of uronic acid metabolism. Biochimica et biophysica acta 30, 186-187.

Bos, C. J., Slakhorst, S. M., Visser, J. \& Roberts, C. F. (1981). A third unlinked gene controlling the pyruvate dehydrogenase complex in Aspergillus nidulans. Journal of Bacteriology 148, 594-599.

Chang, Y. F. \& Feingold, D. S. (1969). Hexuronic acid dehydrogenase of Agrobacterium tumefaciens. Journal of Bacteriology 99, 667-673.

Clutterbuck, A. J. (1973). Gene symbols in Aspergillus nidulans. Genetical Research 21, 291-296.

Cozzarelli, N. R., Koch, J. P., Hayashi, S. \& Lin, E. C. C. (1965). Growth stasis by accumulated L- $\alpha-$ glycerophosphate in Escherichia coli. Journal of Bacteriology 90, 1325-1329.

Ehrlich, F. \& Guttmann, R. (1934). Zür Kenntis der 5-keto-1-galactonsäure (d-Tagaturonsäure). Bericht der Deutsche chemischen Gesellschaft 67, 1345-1347.

Hucho, F., Randall, D. D., Roche, T. E., Burgett, M. W., Pelley, J. W. \& Reed, L. J. (1972). $\alpha$-Keto acid dehydrogenase complexes. XVII. Kinetic and regulatory properties of pyruvate dehydrogenase kinase and pyruvate dehydrogenase phosphatase from bovine kidney and heart. Archives of Biochemistry and Biophysics 151, 328-340.

KenNeY, W. C., Zakim, D., Hogue, P. K. \& Singer, T. P. (1972). Multiplicity and origin of isoenzymes of lipoyl dehydrogenase. European Journal of Biochemistry. 28, 253-260.

Kilgore, W. W. \& Starr, M. P. (1959). Uronate oxidation by phytopathogenic Pseudomonas. Nature, London 183, 1412-1413.

McCullough, W., Payton, M. A. \& Roberts, C. F.
(1977). Carbon metabolism in Aspergillus nidulans. In Genetics and Physiology of Aspergillus, pp. 97-129. Edited by J. E. Smith \& J. A. Pateman. London: Academic Press.

MCCully, K. \& Forbes, E. (1965). The use of $p$ fluorophenylalanine with 'master strains' of Aspergillus nidulans for assigning genes to linkage groups. Genetical Research 6, 352-359.

PAyton, M. A. (1978). A genetic study of sugar metabolism and transport in Aspergillus nidulans. $\mathrm{PhD}$ thesis, University of Leicester, UK.

Payton, M. A., McCullough, W., Roberts, C. F. \& Guest, J. R. (1977). Two unlinked genes for the pyruvate dehydrogenase complex in Aspergillus nidulans. Journal of Bacteriology 129, 1222-1226.

PERKINS, D. D. (1962). Preservation of Neurospora stock cultures with dehydrous silica gel. Canadian Journal of Microbiology 8, 591-594.

Pontecorvo, G., Roper, J. A., Hemmons, L. J., MCDonald, K. D. \& Bufton, A. W. J. (1953). The genetics of Aspergillus nidulans. Advances in Genetics 5, 141-238.

Pratt, J. W. \& Richtmeyer, N. K. (1955). Transformation of D-allose to 1.6-anhydro- $\alpha$-D-allopyranose in acid solution. Journal of the American Chemical Society 77, 1906-1908.

Preiss, J. \& Ashwell, G. (1963a). Polygalacturonic acid metabolism in bacteria. I. Enzymatic formation of 4-deoxy-L-threo-5-hexoseulose uronic acid. Journal of Biological Chemistry 238, 1571-1576.

Preiss, J. \& Ashwell, G. (1963b). Polygalacturonic acid metabolism in bacteria. II. Formation and metabolism of 3-deoxy-D-glycero-2,5-hexodiulosonic acid. Journal of Biological Chemistry 238, 1577-1583.

Sakurai, Y., FukuYoski, Y., Hamada, M., Hayakwa, T. \& KoIKE, M. (1970). Mammalian $\alpha$-ketoacid dehydrogenase complexes. VI. Nature of the multiple forms of pig heart lipoamide dehydrogenase. Journal of Biological Chemistry 245, 4453-4462.

SkinNer, V. M. \& ARmitT, S. (1972). Mutants of Aspergillus nidulans lacking pyruvate carboxylase. FEBS Letters 20, 16-18.

Uitzetter, J. H. A. A., Bos, C. J. \& Visser, J. (1982). Growth characteristics of Aspergillus nidulans mutants defective carbohydrate metabolism. Antonie van Leeuwenhoek 48, 219-227. 